souhaits de réussite accompagneront aussi les essais de M. l'-Ingénieur Defosse. Puissent-ils constituer à son heure l'acte d'émancipation de la " houille grise ».

Mais pourquoi " houille grise"? - Ce mot, nous l'avions déjà entendu et lu; d'aucuns l'emploient pour désigner l'énergie élecurique parcourant les réseaux qu'alimentent à la fois des stations centrales à vapeur et des usines hydrauliques (mélange de houille noire et de houille blanche qui tourne forcément au gris!), réseaux comme il s'en fait de plus en plus.

Nous comprenons très bien qu"on donne un nouveau nom à la force des marées; c'est une modalité sans doute prochainement industrielle de l'Energie; et, ainsi considérée, elle se présente comme une forme de houille très différente naturellement de la forme charbon, et non moins différente aussi de la forme " houille blanche ". En effer, la houille blanche est l'énergie dynamique en ouvre dans un cours d'eau, lequel est le récepteur mécanique dans la machine thermique que constituent un appareil d'évaporation (la mer et le soleil) et un bassin de condensation (les montagnes). Ce qu'on appelle la force des marées n'est due en rien à un phénomène thermodynamique, et sa cause est uniquement un phénomène astronomique. Une différence, aussi essentielle, légitime des appellations différentes. Mais, encore une fois, pourquoi faire de cette force la « houille grise $n$ ? Cela sent les brouillards du Nord. La poésie ne fait rien à l'affaire, mais, à choisir entre deux néologismes, pourquoi ne pas prendre celui qui évoque l'un des plus beaux spectacles de la nature. La mer est bleue, voyons! Qu'on nous parle de « houille bleue ». Le mot, il est vrai, a déjà été dit (poétiquement, certes, mais non rationnellement) à propos de l'énergie potentielle que renferment les flots bleus des hauts lacs alpins. Mais son auteur ne m'en voudra pas de changer sa destination.

Et, ainsi que l'on voit la houille noire et la houille blanche s'unir pour donner de la houille grise, l'on verra la houille verte s'allier à la houille bleue pour faire... Je n'y suis plus! j'en perds la vue.

$$
\text { E.-F. Côte. }
$$

\section{Sur les Effets de Résonance dans les coups de Béliep pour le cas des hautes chutes}

(Suite)

J'ai, dans le numéro de septembre de La Houille Blanche, examiné un cas hypothétique où, pour les hautes chutes, les effets de résonnance peuvent, pour des fermetures brusques, faire prendre au coup de bélier une valeur légèrement supérieure à la pression statique.

Je remarquerai d'abord que ces conclusions subsistent sans aucun changement si, au lieu de fermer ou d'ouvrir brusquement la distribution en plein, au début de chaque période de longueur égale à $2 \frac{l}{a}\left(^{*}\right)$, l'on fermait où l'on ouvrait ce distributeur progressivement en un temps au plus égal à $2 \frac{l}{a}$, à condition toutefois de considérer la valeur du coup de bélier seulement à la fin de chaque période; sans s'occuper de sa valeur pendant les instants intermédiaires. Ceci résulte immédiatement de ce que la valeur du coup de bélier, près du distributeur, dépend seulement de la vitesse de l'eau dans la conduite en ce point, aux instants correspondants des périodes précédentes, c'est-à-dire aux instantts :

$$
t, \quad t-2 \frac{a}{l}, \quad t-4 \frac{l}{a} \quad \text { etc. }
$$

( $)$ l étant la longneur de la conduite et a la vltesse de propagation.
Je me propose aujourd'hui d'examiner le cas où l'orifice de distribution est successivement fermé ou ouvert progressivement dans un temps égal à $\frac{2 l}{a}$, de façon à réduire son ouverture, lorsqu'on la ferme, d'une fraction $\frac{1}{p}$ et à l'ouvrir ensuite en plein pendant la période suivante, mais toujours d'une façon progressive.

Nous verrons que, dans ce cas, les effets de résonnanee peuvent faire prendre au coup de bélier une valeur égale et mêne un peu supérieure a $\frac{\mathcal{Y}_{0}}{p}, \mathcal{Y}_{0}$ désignant toujours la pression statique ${ }^{*}$ ).

Nous considérerons toujours une série de périodes successives de durée égale à $2 \frac{l}{a}$, et je désigne : par $\xi_{n}$ la valeur du coup de bélier à un instant quelconque de la $n^{\mathrm{e}}$ période où l'on a :

$$
2(n-1) \frac{l}{a} \leqslant t \leqslant 2 n \frac{l}{a}
$$

Soit $\lambda_{n}$ le rapport de l'ouverture du distributeur à cet instant à son ouverture à l'état de régime, et $\nu$ la vitesse de l'eau dans la conduite à l'instant $t, \nu_{0}$ étant la vitesse à l'état de régime. Soient de plus, $\xi_{n-1}, \xi_{n-2 . .} \xi_{1}, \lambda_{n-1}$, $\lambda_{n}-2 \ldots \lambda$, les valeurs de $\xi$ et de $\lambda$ a l'instant correspondant de l'une des périodes précédentes, c'est-à-dire pour les valeurs du temps $t-2 \frac{l}{a}, t-4 \frac{l}{a} \ldots t-2(n-\mathrm{I}) \frac{l}{a}$.

'J'ai fait voir $\left(^{* *}\right)$ que si le coup de bélier ne dépasse pas la moitié environ de la pression statique on a entre les valeurs $\xi_{n}$ et $\xi_{n-1}$ du coup de bélier, à deux instants correspondants de la $n^{\mathrm{e}}$ et de la $(n-\mathrm{I})^{\mathrm{e}}$ période, les relations :

$$
\xi_{n}=\frac{\frac{a \nu_{0}}{g}\left(\lambda_{n-1}-\lambda_{n}\right)}{\mathrm{I}+\frac{a \nu_{0}}{2 g \gamma_{0}} \lambda_{n}}-\xi_{n-1} \frac{1-\frac{a \nu_{0}}{2 g \gamma_{0}} \lambda_{n-1}}{1+\frac{a \nu_{0}}{2 g \gamma_{0}} \lambda_{n}}
$$

où $g$ désigne l'accélération de la pesanteur et $y_{0}$ la pression statique.

De plus, dans les mêmes conditions, le coup de bélier à l'instant correspondant de la prèmière période est donné par la formule :

$$
\xi_{1}=\frac{\frac{a \nu_{0}}{g}\left(1-\lambda_{1}\right)}{1+\frac{a \nu_{0}}{2 g \gamma_{0}} \lambda_{1}}
$$

Supposons maintenant que l'on ferme l'orifice du distributeur d'une façon progressive pendant la première période, de manière à réduire sun ouverture à la fin de cette période de la fraction $\frac{\mathbf{I}}{p}$.

Pendant la période suivante, on l'ouvrira progressivement, de façon qu'il soit complètement ouvert à la fin de cette

${ }^{\star}$ ) Les formules d'où nous partirons supposent toutefois que $p$ n'est pas inférieure à 2 .

$\left.{ }^{\star \star}\right)$ Etude théorique sur les coups de bélier dans les conduites forcées (voir La Houille Blanche de juillet Igoj, page 160). 
période. Pendant la $3 e$ période on la refermera progressivement de nouveau de la fraction $\frac{\mathrm{I}}{p}$, comme pendant la première période; puis, pendant la quatrième période, on l'ouvrira de nouveau progressivement comme pendant la seconde, et ainsi de suite.

Considérons alors la $(2 n+1)^{e}$ période où $t$ varie de $4 n \frac{l}{a}$ à $2(2 n+1) \frac{l}{a}$ et où l'ouverture est progressivement fermé de $\frac{1}{p}$.

Posons $t=\tau+4 n \frac{l}{a}$

$\tau$ variant donc pendant cette période de o à $2 \frac{l}{a}$ :

Nous aurons, d'après ce qui a été dit, la fermeture étant supposée uniforme:

$$
\lambda q_{R}+1=1-\frac{\tau a}{2 l p}
$$

Nous aurons de même, pour les instants correspondants des périodes précédentes.

$$
\begin{aligned}
& \lambda_{2 n}=\mathrm{I}-\frac{\mathrm{I}}{p}+\frac{a \tau}{2 l p} \\
& \lambda_{2 n-1}=\lambda_{2 n+1}=\mathrm{I}-\frac{\tau a}{2 l p}
\end{aligned}
$$

On aura donc :

$$
\lambda_{2 n-1}-\lambda_{2 n}=-\left(\frac{a \tau}{l p}-\frac{1}{p}\right)=-\left(\lambda_{2 n}-\lambda_{2 n}+1\right)
$$

Si, par suite, dans la formule ( 1 ), nous faisons successivement $n$ égal à $2 n+1$ et à $2 n$, et que nous chassions les dénominateurs, nous aurons :

$$
\begin{aligned}
& \left(\mathrm{I}+\frac{a \nu_{0}}{2 g y_{0}} \lambda_{2 n}+1\right) \xi_{2 n}+1=\frac{a \nu_{0}}{g}\left(\frac{a \tau}{l p}-\frac{\mathrm{I}}{p}\right)-\xi_{2 n}\left(\mathrm{I}-\frac{a \nu_{0}}{2 g y_{0}} \lambda_{2 n}\right) \\
& \left(\mathrm{I}+\frac{a \nu_{0}}{2 g y_{0}} \lambda_{2 n}\right) \xi_{2 n}=-\frac{a \mu_{0}}{g}\left(\frac{a \tau}{l p}-\frac{1}{p}\right)-\xi_{2 n}-1\left(\mathrm{I}-\frac{a \nu_{0}}{2 g \gamma_{0}} \lambda_{2 n}-1\right)
\end{aligned}
$$

On en deduit $\left(^{*}\right)$ :

$\mathrm{Z}_{\xi 2 n+1}=\frac{2 a r_{0}}{g}\left(\frac{a \tau}{l_{p}}-\frac{\mathrm{I}}{p}\right)+\left(1-\frac{a \gamma_{0}}{2 g \gamma_{0}} \lambda_{\Omega_{n}}\right)\left(1-\frac{a \nu_{0}}{2 g y_{0}} \lambda_{2 n+1}\right) \xi_{2 n-1}$

avec :

$$
\mathrm{Z}=\left(\mathrm{I}+\frac{a \gamma_{0}}{2 g \gamma_{0}} \lambda_{2 n+1}\right)\left(\mathrm{I}+\frac{a \nu_{0}}{2 g \gamma_{0}} \lambda_{2 n}\right)
$$

Posons maintenant :

$$
\xi_{2 n+1}=\alpha+\xi_{2 n}+1
$$

où $\alpha$ est indépendant de $n$, de sorte que l'on aura aussi :

Nous aurons :

$$
\xi_{2 n-1}=\alpha+\zeta_{2 n-1}
$$

$$
\mathrm{Z}\left\lfloor a_{n+1}=\left\{\begin{array}{l}
\left(\mathrm{I}-\frac{a \nu_{0}}{2 g y_{0}} \lambda_{2 n+1}\right)\left(\mathrm{I}-\frac{a \nu_{0}}{2 g y_{0}} \lambda_{2 n}\right) \zeta_{2 n-1} \\
+\frac{2 a \nu_{0}}{g}\left(\frac{a \tau}{l p}-\frac{1}{p}\right)-\frac{2 a \nu_{0}}{g y_{0}}\left(1-\frac{1}{2 p}\right) \alpha
\end{array}\right\}\right.
$$

(*) Puisque $\lambda_{2 n}-1=\lambda_{2 n}+1$
Nous déduisons, en effet, de (4) et de (5):

$$
\begin{aligned}
& \left.\left(\mathrm{I}+\frac{a \nu_{0}}{2 g \gamma_{0}} \lambda_{\Omega_{n+1}}\right)\left(\mathrm{I}+\frac{a \nu_{0}}{2 g \gamma_{0}} \lambda_{Q_{n}}\right)\right)=\frac{2 a \nu_{0}}{g \gamma_{0}}\left(\mathrm{I}-\frac{\mathrm{I}}{2 p}\right) \\
& \left.-\left(\mathrm{I}-\frac{a \nu_{0}}{2 g y_{0}} \lambda_{a_{n+1}}\right)\left(\mathrm{I}-\frac{a \nu_{0}}{2 g y_{0}} \lambda_{a_{n}}\right)\right\}=\frac{a \nu_{0}}{g y_{0}}\left(\lambda_{\Omega_{n+1}}+\lambda_{n}\right)
\end{aligned}
$$

Si alors nous prenons :

$$
\alpha=y_{0} \frac{\frac{a \tau}{l}-\mathrm{I}}{p-\frac{1}{2}}
$$

Nous aurons :

$$
\xi_{0 n-1}=\mu, \xi_{2 n-1}
$$

avec :

$$
\mu=\frac{\left(1-\frac{a \nu_{0}}{2 g y_{0}} \lambda_{2 n+1}\right)\left(1-\frac{a \nu_{0}}{2 g y_{0}} \lambda_{2 n}\right)}{\left(1+\frac{a \nu_{0}}{2 g y_{0}} \lambda_{2 n}+1\right)\left(1+\frac{a \nu_{0}}{2 g y_{0}} \lambda\right)}
$$

c'est-à-dire, en tenant compte des valeurs $\lambda_{g_{n}}-1$ et $\lambda_{2 n}$ :

$$
\mu=\frac{\left[1-\frac{a \nu_{0}}{2 g y_{0}}\left(1-\frac{\tau a}{2 l p}\right)\right]\left[1-\frac{a \nu_{0}}{2 g \gamma_{0}}\left(1-\frac{\mathrm{I}}{p}+\frac{\tau a}{2 l p}\right)\right]}{\left[\mathrm{I}+\frac{a \nu_{0}}{2 g y_{0}}\left(1-\frac{\tau a}{2 l p}\right)\right]\left[1+\frac{a \nu_{0}}{2 g y_{0}}\left(1-\frac{1}{p}+\frac{\tau a}{2 l p}\right)\right]}
$$

et l'application successive de la formule (8) donnera :

$$
\zeta_{2 n+1}=\mu^{n} \zeta_{1},
$$

Si alors on tient compte de la relation :

$$
\xi_{2 n+1}=\alpha+\zeta_{2 n+1}
$$

Nous aurons, pour la valeur du coup de bélier pendant la $(2 n+1)$ e période :

$$
\xi_{2 n+1}=\alpha-\mu^{n}\left(\alpha-\xi_{1}\right)
$$

D'ailleurs, si on remplace $\lambda_{1}$ par sa valeur, la formule (2) donne :

$$
\xi_{1}=\frac{\frac{a^{2} \tau \nu_{0}}{2 p g l}}{1+\frac{a \nu_{0}}{2 g y_{0}}\left(1-\frac{a \tau}{2 p l}\right)}
$$

formules dans lesquelles $\tau$ varie de o à $\frac{2 l}{a}$.

La formule (i I) fait vorr que, si l'on considère un nombre d'oscillations de plus en plus considérable, le coup de bélier tend à augmenter jusqu'à la valeur $\alpha$. Il faut remarquer, toutefois, que ceci n'est exact que si l'on a :

$$
\alpha \leqslant \frac{y_{0}}{2}
$$

puisque les formules dont nous sommes partis supposent que le coup de bélier ne dépasse pas environ la moitié de la charge statique.

Proposons nous en particulier de calculer ce que seront les coups de bélier à la fin de chacune des périodes de rang impair ce qui correspond à leuŕs maxima, nous aurons ainsi les formules suivantes qui sont celles dont il y a lieu de faire usage pour les applications. 
Donc, pour $\tau=2 \frac{l}{a}$, et en désignant par par $\xi^{\prime}, \alpha^{\prime}, \zeta^{\prime}, \mu^{\prime}$ les valeurs correspondantes de $\xi, \alpha, \zeta, \mu$.

$$
\begin{gathered}
\xi_{1}^{\prime}=\frac{\frac{a \nu_{0}}{p g}}{\mathrm{I}+\frac{a \nu_{0}}{2 g y^{0}}\left(\mathrm{I}-\frac{\mathrm{I}}{p}\right)} \\
\alpha^{\prime}=\frac{2 y_{0}}{2 p-1} \\
\mu_{1}^{\prime}=\frac{\xi_{1}^{\prime}=\xi_{1}^{\prime}-\alpha^{\prime}}{\left[\mathrm{I}-\frac{a \nu_{0}}{2 g y_{0}}\left(\mathrm{I}-\frac{1}{p}\right)\right]\left(\mathrm{I}-\frac{a \nu_{0}}{2 g y_{0}}\right)} \\
{\left[1+\frac{a \nu_{0}}{2 g y_{0}}\left(\mathrm{I}-\frac{\mathrm{I}}{p}\right)\right]\left(\mathrm{I}+\frac{a \nu_{0}}{2 g y_{0}}\right)} \\
\zeta_{2 n+1}^{\prime}=\mathrm{n}^{\prime} \zeta_{2 n-1}^{\prime}=\mu^{\prime} \zeta_{1} \\
\xi_{2 n+1}^{\prime}=x^{\prime}+\zeta_{2 n+1}^{\prime}
\end{gathered}
$$

Ces formules, toutefois, ne doivent être employées, d'après ce que nous avons dit, que tant que l'on a :

$$
\xi_{2 n+1}^{\prime} \leqslant \frac{\gamma_{0}}{2} \text {. }
$$

Comme p.' est toujours plus petit que I, la formule ( 8 ) fait voir que $\zeta_{2 n+1}^{\prime}$ a pour limite zéro lorsque $n$ croit indéfiniment, et que, par suite, le coup de bélier $\xi_{g n+1}^{\prime}$ a pour limite $\alpha$.

Si nous considérons la valeur de $\alpha$, elle pourra s'écrire :

$$
\alpha^{\prime}=\frac{y_{0}}{p}\left(\frac{1}{1-\frac{1}{2 p}}\right)
$$

et cette expression fait voir que a' est plus grand que $\frac{y_{0}}{p}$ mais, comme $p$ doit être supérieurà 2 , le terme $\left(\frac{1}{1-\frac{1}{2 p}}\right)$ est inférieur à $\frac{4}{3}$,et l'on peut dire approximativement, ainsi que nous l'avons annoncé. que si l'ouverture du distributeur est réduite de $\frac{1}{p}$ dans les conditions que nous avons indiquées, le coup de bélier peut, par suite des résonnances; devenir sensiblement égal à $\frac{y_{0}}{p}$.

Comme application, supposons que l'on ait :

Nous aurons:

$$
y_{0}=500 \mathrm{~m} . \quad \frac{a \nu_{0}}{g}=100 \mathrm{~m} .
$$

$$
\begin{array}{cl}
\xi_{1}^{\prime}=\frac{1000}{11 p-1}- & \alpha_{1}^{\prime}=\frac{1000}{2 p-1} \\
\xi_{1}^{\prime}=\frac{-9000 p}{(2 p-1)(11 p-I)} & \mu^{\prime}=\frac{9(9 p+1)}{11(11 p-1)} .
\end{array}
$$

Si l'on prend alors $p=2$, on aura :

$$
\alpha_{1}^{\prime}=333,3 ; \quad \zeta_{1}^{\prime}=-285,7 ; \quad \mu^{\prime}=0,74 ; \quad \xi_{1}=47,6
$$

et on aura pour les coups de bélier successifs :

$$
\xi_{3}=122 ; \xi_{5}=\mathrm{J} 77 ; \xi_{7}=218 ; \xi_{9}=248 ;
$$

donc $\xi_{9}$ est déjà sensiblement égal à $\frac{y_{0}}{2}$.
Prenons ensuite $p=5$, nous aurons:

$$
\alpha_{1}^{\prime}=111 ; \zeta_{1}^{\prime}=-92,6 ; \mu{ }^{\prime}=0,7 ; \xi_{1}^{\prime}=18,5 ;
$$

et les coups de bélier successifs deviendront:

$$
\xi_{3}=46 ; \xi_{5}=66 ; \xi_{7}=80 ; \xi_{9}=89 ;
$$

Prenons enfin $p=10$, nous aurons:

$$
\alpha_{1}^{\prime}=52,6 ; \zeta_{1}^{\prime}=-43,4 ; \mu^{\prime}=0,68 ; \xi_{1}^{\prime}=9,2 ;
$$

et nous en déduirons pour les coups de bélier successifs :

$$
\xi_{3}^{\prime}=24 ; \xi_{5}^{\prime}=33 ; \xi_{7}^{\prime}=39 ; \xi_{9}^{\prime}=43 .
$$

On voit, par ces exemples, que dans le cas d'une fermeture partielle réduisant l'ouverture du distributeur de $\frac{\mathrm{I}}{p}$, le coup de bélier devient assez rapidement sensiblement égal à $\frac{y_{0}}{p}, y_{0}$ désignant la charge statique, valeur qui, si $y_{0}$ est considérable, pourra être très supérieure au coup de bélier, comme dans le premier exemple que nous avons donné, qui correspond à une fermeture totale brusque sans effet de résonnance.

Nous remarquerons aussi que, si pour obtenir la valeur du coup de bélier à un instant quelconque, nous avons supposé une fermeture uniforme, cette hypothèse de la fermeture uniforme n'interviendrait absolument en rien si on se bornait à chercher la valeur ' $\xi$ ' du coup de bélier à la fin de chaque période. Il suffit, pour le voir, de se reporter à ce que nous avons dit en débutant pour le cas de la ferme. ture totale.

\section{Comte de Sparre, Doyen de la Faculté libre des Sciences de Lyon.}

\section{Décret du 17 Octobre 1907, organisant le service du Contrôle des Distributions d'énergie électrique}

Le. Président de la République française,

Sur le rapport du ministre de l'intérieur, du ministre des travaux publics, des postes et des télégraphes, et du ministre de l'agricalture,

Vu la loi du i 5 juin r 906 sur les distributions d'énergie, et notamment l'article $18\left(3^{\circ}\right)$ portant qu'un règlement d'administration publique détermine l'organisation du contrôle de la construction et de l'exploitation des distributions d'énergie électrique ;

Vu l'avis du ministre des finances en date du 3 r juillet 1907 , Le Conseil d'Etat entendu,

\section{Décrète :}

I. - Distributions établies en vértu de concessions accordées par L'ETAT, ET DISTRIBUTIONS IMMRUNTANT EN TOUT OU EN PARTIE LA GRANDE VOIRIE EN VERTU DE PERMISSIONS.

Article premier. - Le contrôle des distributions d'énergie électrique établies en vertu de concessions accordées par l'Etat et des distributions empruntant en tout ou en partie la grande voirie en vertu de permissions, est exercé dans chaque département par un ingénieur en chef.

Deux ou plusieurs départements peuvent, par decision spéciale du ministre des travaux publics, être réunis en une circonscription unique.

Art. 2. - L'ingénieur en chef du contrôle des distributions d'énergie électrique est assisté d'agents dont le nombre et la 